\title{
Balancing the Size-Number Tradeoff in Clonal Broods
}

\author{
Philip H. Crowley ${ }^{*}$ and Yoriko Saeki
}

Department of Biology and Center for Ecology, Evolution \& Behavior, University of Kentucky, Lexington, KY 405060225, USA

\begin{abstract}
The trade-off between the number of offspring in a brood and the sizes of those offspring has been documented in a diverse array of species. Here we consider the factors that might account for the way that the trade-off achieves a particular size-number balance for female offspring. In particular, we determine whether bet hedging, along with traits influencing the expected short-term reproductive success of the brood, could select for a brood size and body mass that maximize long-term fitness. We also evaluate whether the optima based on these characteristics agree qualitatively with documented responses to brood resource levels. To develop a model incorporating these phenomena and keep it as simple and general as possible, we consider an organism that produces clonal broods, eliminating the complexities associated with parent-offspring and sibling conflict. We use the polyembryonic parasitoid wasp Copidosoma bakeri as the focal example. We find that bet hedging effects and key reproductive traits (the number of searching females, their host-finding efficiency, and survival prospects) are often sufficient to produce an optimal size-number balance and can account for the frequent tendency of both brood size and body mass to increase with brood resources (host size). Under some conditions, however, balancing the trade-off requires an additional minimum body-size constraint. Future empirical work and spatially explicit models must better establish the parameter magnitudes and functional relationships so that a deeper understanding and more precise predictions are obtained.
\end{abstract}

Keywords: Bet hedging, body size, brood size, clutch size, mathematical modeling, optimal life history, parasitoid wasps, polyembryony.

\section{INTRODUCTION}

The life-history concept of a best or optimal brood size began with Lack's (1947) studies of bird clutches. A great many studies of birds and other vertebrates have followed (see Stearns 1992, Roff 2002), often based on the tacit assumption that body mass at birth reflects adaptation to both intra-brood competition and the post-natal environment, while clutch or brood size (offspring number) expresses an optimal reproductive strategy by parents, given this offspring body mass. Thus attempts to understand body mass and brood size have often proceeded independently, but there are deeper insights to be gained from considering them together (Mayhew and Glaizot 2001). Studies of invertebrates have often suggested that offspring production expresses a sizenumber tradeoff (Godfray 1994, Roff 2002). Especially in females, larger sizes of individual offspring, typically associated with greater fecundity, trade off against the greater number of offspring that could be produced if offspring were smaller. Understanding how this trade-off gets balanced between size and number is particularly challenging because of the range of selection pressures expected to act on both body size and brood number of offspring from early development through adulthood.

Sibling competition (e.g. see Hardy et al. 1992, Traynor and Mayhew 2005) and parent-offspring conflict (Godfray

*Address correspondence to this author at the Department of Biology and Center for Ecology, Evolution \& Behavior, University of Kentucky, Lexington, KY 40506-0225, USA; Tel: 859-257-1996, Fax: 859-257-1717; E-mail: pcrowley@uky.edu

Handling Editor: Volker Grimm
1994) are often intense in genetically mixed broods. But for the special case of clonal broods, the size-number trade-off seems to become conceptually simpler (Fig. 1). Members of the brood are no longer in competition in the sense of fitness costs; this is because the resources available to determine brood mass are presumably distributed among separate individuals under selection to maximize the ultimate reproductive success of the brood as a whole (see Godfray 1987, Godfray and Parker 1991, and Godfray 1994). This distribution among individual offspring is not subject to parent-offspring conflict, since parental fitness also hinges on reproductive success of the whole brood, though the amount of resources provided to the brood may be a source of conflict when current reproduction restricts future reproduction of the parent.

But even this potential conflict over maternal brood resources disappears in the case of polyembryonic wasps with clonal broods. Here the small maternal investment of a single yolkless egg oviposited into a host results in repeated cell divisions and ultimately a large group of clonal individuals developing at the expense of the host's tissues (Ivanova-Kasas 1972). As with other parasitic wasps, selection on development time is unlikely to constrain either body mass or brood number (Mayhew and Glaizot 2001; cf. body mass vs development time in Crowley 2000). In this instructive clonal-brood situation, where resolution of the size-number tradeoff depends primarily or entirely on selection pressures acting on adults arising from the brood, how are size and number determined? How might an optimal balance be achieved? 


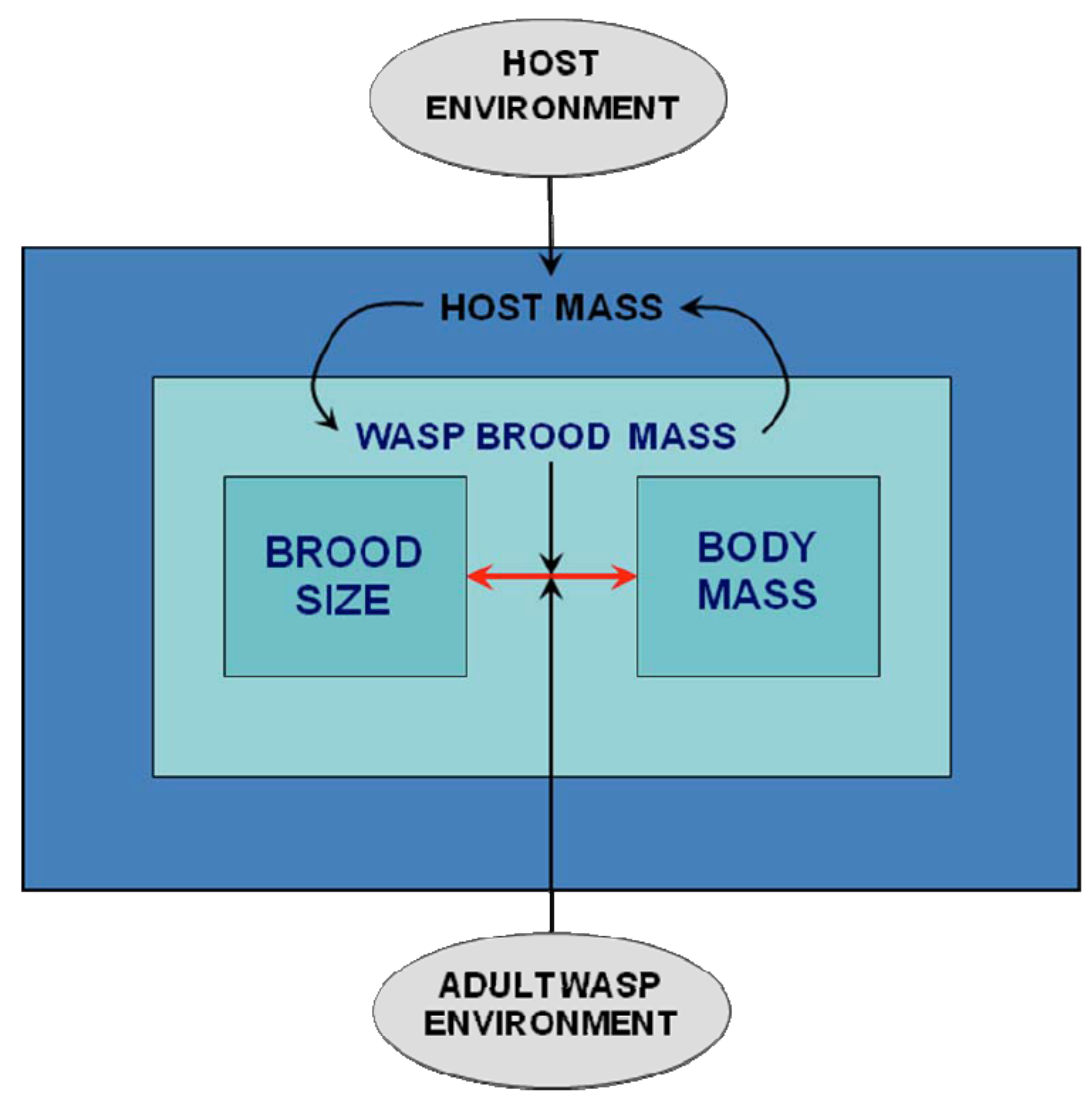

Fig. (1). Diagrammatic representation of the size-number tradeoff in clonal broods. The environment of the host during its development strongly influences the ultimate host mass, which represents the resources available to the wasp brood that determine brood mass at wasp emergence. (The host mass can also be influenced by the developing wasp brood--see Saeki et al. 2009.) Within the wasp brood there is a size-number trade-off between brood size and body mass, influenced by wasp brood mass and strongly reflecting selection pressures acting in the adult wasp environment. In non-clonal broods, brood mass and the size-number tradeoff also depend on intra-brood competition, parent-offspring conflict, and sex ratio within the brood in addition to selection pressures on the adult stage.

Simple models of optimal clutch or brood size indicate that the body mass (and thus the fitness) of individual offspring should be independent of the resources available to the developing brood-more resources should simply generate more offspring of the same body mass (e.g. see Smith and Fretwell 1974, Parker and Courtney 1984, Waage and Godfray 1985). Yet in some cases, both brood size and body mass increase with available resources (e.g. both may increase with host mass in parasitic wasps, especially among female offspring — see data in Hardy et al. 1992 and Mayhew and Hardy 1998), though models capable of explaining this are rare (but see Mayhew and Glaizot 2001). Here we consider specifically how body mass and brood number might be expected to depend on host size for clonal female broods of a polyembryonic wasp. We focus on this scenario by virtue of its relative simplicity and thus its potential for empirical study - but also because it introduces some issues of general importance and casts some features of genetically diverse broods in sharp relief.

\section{CONSTRUCTING AND ANALYZING A MODEL}

To address this question, we focus on how natural selection should influence female broods, under the assumption that body mass in male broods is largely determined by sexual selection for compatibility with female body mass.
We then imagine that the problem for a clonal female brood is to produce the optimal number and size of females to maximize the lifetime total number of hosts into which they can oviposit. Since we assume brood mass (the mathematical product of brood size and mean wasp mass) to be set by the resources contained in the eventual host mass, it follows that the number and size of these ovipositing agents are inversely related for a given host mass. Note that balancing the sizenumber trade-off is apparently addressed by the developing brood itself rather than the ovipositing female, who provided only a single egg at a time when the resources ultimately available to offspring would be difficult or impossible to predict (Godfray 1994).

To keep things manageably simple, we assume that eggs are inexpensive (and thus unlimited) relative to searching for hosts, which, though superabundant, are time-consuming to locate (i.e. host or time limitation rather than egg limitation). We further assume that independent searching for superabundant hosts precludes superparasitism. Moreover, selection on female body size in the post-emergence environment, along with the limited variation expected to arise from the single developmental program and host environment, should produce emerging adults very similar in size. We therefore focus on a single body mass assumed to characterize all individuals in a brood. Variables and parameters of the model are listed in Table $\mathbf{1}$. 
Table 1. Parameters and Variables of the Model ${ }^{1}$

\begin{tabular}{|c|c|c|c|}
\hline Symbol & Definition & Magnitude(s) ${ }^{1}$ & Units \\
\hline$b$ & Constant of proportionality: brood's squared coefficient of variation, eq. (4) & Free & Units of $n^{y-x} h^{-y}$ \\
\hline$c$ & Coefficient of variation for individual reproductive success, eq. (3) & Arbitrary & None \\
\hline$C$ & Coefficient of variation for brood reproductive success, eq. (4) & Variable & None \\
\hline$h / n$ & Body mass of an individual wasp, eq. (4) & Variable & Mass individual $^{-1}$ \\
\hline$h / \hat{n}$ & Optimal body mass of an individual wasp, eq. (7) & Variable & Mass individual $^{-1}$ \\
\hline$i$ & Number of subdivisions of a brood that reproduce independently & $1<i<n$ & None \\
\hline$k$ & Constant of proportionality for $m(n, h)$, where $k=k_{l} k_{2} k_{3}$, eq. (1) & Arbitrary & Units of $h^{-a} n^{a-1}$ \\
\hline$n$ & Brood size: number of emerging wasps in a brood, eqs. (1), (4), and (5) & Variable & Individuals \\
\hline$\hat{n}$ & Optimal brood size, eqs. (6) and (7) & Variable & Individuals \\
\hline$u$ & Exponent of power function: adult survival time, Fig. (2) and eq. (1) & Arbitrary & None \\
\hline$v$ & Long-term (lineage) variance in individual reproductive success & Arbitrary & None \\
\hline$w$ & Expected lineage fitness, eqs. (2), (3), and (5) & Variable & None \\
\hline$x$ & Power function exponent: reproductive independence $v s$. brood size, eq. (4) & -0.5 to 0 & None \\
\hline$y$ & Power function exponent: reproductive independence $v s$. body mass, eq. (4) & -0.3 to 0.3 & None \\
\hline$z$ & Power function exponent: parasitization rate vs body mass, Fig. (2), eq. (1) & Arbitrary & None \\
\hline
\end{tabular}

"The parameter $b$ is a "free" parameter in the sense that its magnitude is chosen to yield the most plausible results. The magnitudes of some parameters have no effect on the outcomes of interest ("arbitrary"); those with numbers indicated are estimated from data, or a plausible range of magnitudes is shown based on logic presented in the text.

${ }^{2}$ Units of these three constants of proportionality are, respectively: none, $(h / n)^{-u}$ time, and $(h / n)^{-z}$ time $^{-1}$.

\section{How Fitness Depends on Brood Size and Body Mass}

We begin with a newly parasitized host egg containing a female wasp egg and consider the fitness of the parasitoid to be the expected total number of parasitized host eggs $m$ generated by the wasps emerging from the original parasitized host egg (Fig. 2). For simplicity we assume that females are immediately mated on emergence (but see Crowley et al. 2008 for some complexities that arise at low population densities from distortions of sex ratio with clonal broods). We initially ignore effects of variation in reproductive success between generations to focus on short-term reproductive success $m$. Then we include the long-term fitness costs associated with fitness variation between generations.

Let $H$ represent relative maximum host mass, where host mass is expressed as a ratio to some benchmark magnitude of host mass so that $H$ is dimensionless. Let $h$ represent the relative brood mass (hereafter referred to simply as brood mass), the portion of $H$ that can be turned into wasp biomass. We assume that $h / H$ is a fraction that is independent of host mass, wasp body size, and brood number (e.g. see Saeki et al. 2009). Then relative wasp body mass (hereafter termed simply body mass) is $h / n$, with $n$ being the brood size.

We represent the fitness $m$ as a function of $n$ and the amount $h$ of host mass ultimately available to produce wasp biomass. We address three main effects on $m(n, h)$ : (1) the "parallel-processing" effect, referring to the potential for more total oviposition to be achieved by more individuals simultaneously seeking and laying eggs into discovered hosts; (2) the "exploitation" effect, expressing the generally positive effect of body size on the rate at which individual females find and parasitize hosts (Glenn and Hoffman 1997); and (3) the "vulnerability" effect, in which smaller individuals have higher mortality rates from greater susceptibility to physical damage (e.g. wind, rain, desiccation), though perhaps also influenced by size-dependent predation, generally resulting in longer adult lifetimes for larger wasps.

Following the logic of Fig. (2), $m(n, h)$ is the multiplicative product of: the chance that the brood survives to emergence, assumed in the apparent absence of contrary evidence to be independent of $n$ and $h$; the brood size at emergence $n$ (the parallel-processing effect); and the number of parasitized eggs produced per emerging female, which is in turn the product of the adult survival time (expressing the vulnerability effect) and the parasitization rate (the exploitation effect), both taken to be power functions of wasp body mass $h / n$. Thus

$m(n, h)=k_{1} k_{2} k_{3} n\left(\frac{h}{n}\right)^{u+z}=k n\left(\frac{h}{n}\right)^{a}$,

where $k_{1}, k_{2}, k_{3}, k\left(=k_{1} k_{2} k_{3}\right), u, z$, and $a(=u+z)$ are constants. 


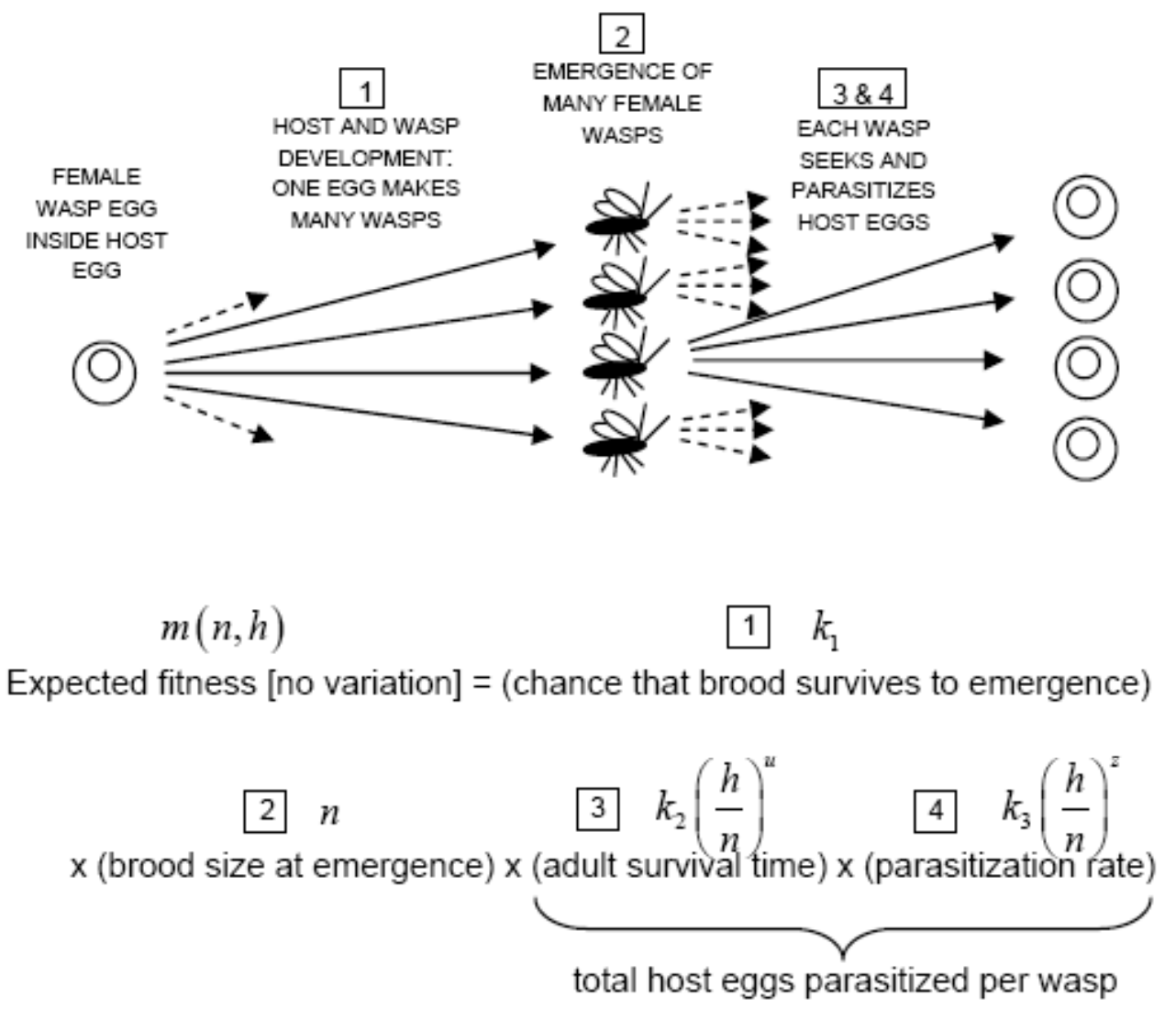

Fig. (2). Beginning with a female wasp egg inside a host egg, the wasp brood must survive and develop to emergence, after which each female seeks and parasitizes host eggs until she dies. Each component of this complete generation can be depicted using simple functions of brood size $n$ and brood mass $h$, providing a model of expected fitness $m(n, h)$ without accounting for generation-to-generation variation. That variation is addressed by the bet-hedging part of the model not shown here.

Note here that whether larger brood size $n$ increases or decreases expected (mean) fitness $m$ depends on the magnitude of $a$ relative to 1 . If $a>1$, exploitation and vulnerability effects dominate parallel processing, giving an advantage to larger body mass over brood size; if $a<1$, parallel processing dominates exploitation and vulnerability, making larger brood size more advantageous than larger body size. These factors and the resulting function alone, however, cannot produce a finite fitness maximum corresponding to optimal brood size $\hat{n}$ and body mass $h / \hat{n}$, except in the sense that $\hat{n}=1$ and body mass $h / \hat{n}$ is $h$ when $a>1$.

\section{Bet Hedging and the Variance of Brood Fitness}

Under generation-to-generation stochastic variation in reproductive success, brood size and body mass may influence long-term fitness through bet hedging. (See Godfray 1994, p. 74ff, and references therein on the application of this concept to parasitoids.) Let $w$ be the long-term fitness of individuals, and let $m$ be the expected short-term fitness. Then $\ln w$ is the per-capita rate of increase per generation, and the expected value of $\ln w$ over many generations can be approximated using the first three terms of a Taylor-series expansion about $m$ :

$$
E(\ln w) \approx E\left(\ln m+\frac{w-m}{m}-\frac{(w-m)^{2}}{2 m^{2}}\right)=\ln m-\frac{v}{2 m^{2}},
$$

where $v$ is the long-term variance in $w$, under the simplifying assumption of statistical independence between generations, and thus by exponentiating both sides of (2)

$$
E(w) \approx m e^{-\frac{c^{2}}{2}}
$$

where $c$ is the coefficient of variation, or $\sqrt{v} / m$ (Lewontin and Cohen 1969, Bulmer 1994, Morris et al. 2008). Note that expected fitness decreases as the coefficient of variation increases.

It is essential to recognize that the squared coefficient of variation in reproductive success over the entire brood, which we symbolize by $C^{2}$, is likely to depend not only on $c^{2}$ for individual offspring but also on brood size and body mass. Parasitoid wasps are known to disperse widely (Askew 1968, Copland and Askew 1997) and may use a larger brood size to reduce $C^{2}$ by achieving more statistical independence in reproductive success within the brood. (For example, if the $n$ individuals in the brood included $i$ subsets that reproduced independently of each other, then, all else equal, the brood variance would be inversely related to $i$ without altering the brood mean, resulting in $C^{2}=c^{2} / i$.) Also, the magnitude of $C^{2}$ may decrease with increasing body mass if larger individuals tend to vary less in reproductive success than smaller ones (Fox and Czesak 2000), or $C^{2}$ may decrease with decreasing body mass if smaller individuals disperse more widely (e.g. by wind) among independently 
varying heterogeneous environments (see related ideas in Crowley 1977). Again using power functions to represent the effects of brood size and body mass, we have

$$
C^{2}=2 b c^{2} n^{x}\left(\frac{h}{n}\right)^{y},
$$

where $b$ is a constant of proportionality, $x$ and $y$ are the brood number and body mass exponents, and the 2 is included to simplify the notation below. (We assume here that $c^{2}$ is evaluated at some constant body mass and note that $c^{2}$, as well $C^{2}$, could depend on body mass. The equation depicts the overall mass-dependency of $C^{2}$ in a way that can also incorporate any mass-dependency of $c^{2}$.)

Equation (4) indicates that fitness is increased by bet hedging (through the reduction of $C^{2}$ ) with $y>x$ when brood size is increased and body mass decreased-but with $x>y$ when brood size is decreased and body mass increased. Substituting equation (1) into (3) and replacing $c^{2}$ in equation (3) by $C^{2}$, we can now express the long-term fitness $w$ as

$$
w(n, h)=k h^{a} n^{1-a} e^{-b c^{2} h^{y} n^{x-y}}
$$

\section{Finding the Optimal Brood Size and Body Mass}

Invoking the simplifying assumption that $n$ can be considered continuous, we find the magnitude of $n$ that maximizes $w, \hat{n}$, by calculating $d w / d n$, setting this to zero, and solving for $\hat{n}$, resulting in

$\hat{n}=\left(\frac{b c^{2}(y-x) h^{y}}{a-1}\right)^{\frac{1}{y-x}}$

It can readily be shown that $d^{2} w / d n^{2}<0$ at $\hat{n}$, and thus that $\hat{n}$ is a maximum, whenever either $y>x$ and $a>1$ or $y<$ $x$ and $a<1$. (Note that $\hat{n}>0$ also requires that these same inequalities be satisfied.) Dividing both sides of equation (6) into $h$ yields the optimal body mass $\frac{h}{\hat{n}}=\left(\frac{b c^{2}(y-x) h^{x}}{a-1}\right)^{\frac{1}{x-y}}$

Fig. (3) illustrates the model outcomes that result from the combination of within-generation fitness effects that depend on the body mass exponent $a$ from equation (1) and the within-brood reproductive independence exponents $x$ and $y$ from equation (4). When magnitudes of the exponents that favor increased body mass are offset by those favoring larger brood size (i.e. when $y>x$ and $a>1$ or $y<x$ and $a<1$ ), the result is the optimal brood size and body mass indicated in equations (6) and (7). When both favor larger body mass (i.e. when $y<x$ and $a>1$ ), the brood consists of a single offspring, and its body mass is $h$. When both favor larger brood size, some additional constraint must set the lower limit of body mass at $(h / n)_{\min }$, resulting in $h / \hat{n}=(h / n)_{\min }$ and $\hat{n}=h /(h / n)_{\min }$.

\section{Estimating Parameter Magnitudes for Copidosoma Bakeri}

Though the host mass available to the parasitoid brood, the brood mass $h$, can be directly determined empirically, estimating the other parameters poses difficulties. Coefficients of variation of individual fitness through time are generally thought to be of order 1 , but very few data bearing on this are available. We focused in the sensitivity analysis on modest deviations around 1 as the best indicator of how the results might depend on an unknown amount of variability in this parameter. Perhaps the variance and mean of individual reproductive success tend to covary in this system such that $c$ remains relatively constant, but we know of no relevant data. The exponent $x$ should generally be negative, since (all else equal) more females means greater spatial spread of oviposition and greater diversity of local conditions conducive to increased statistical independence of reproductive success and lower $C^{2}$. But $x$ should be small in absolute value, consistent with the relatively high correlation to be expected in response to fitness variation by genetically

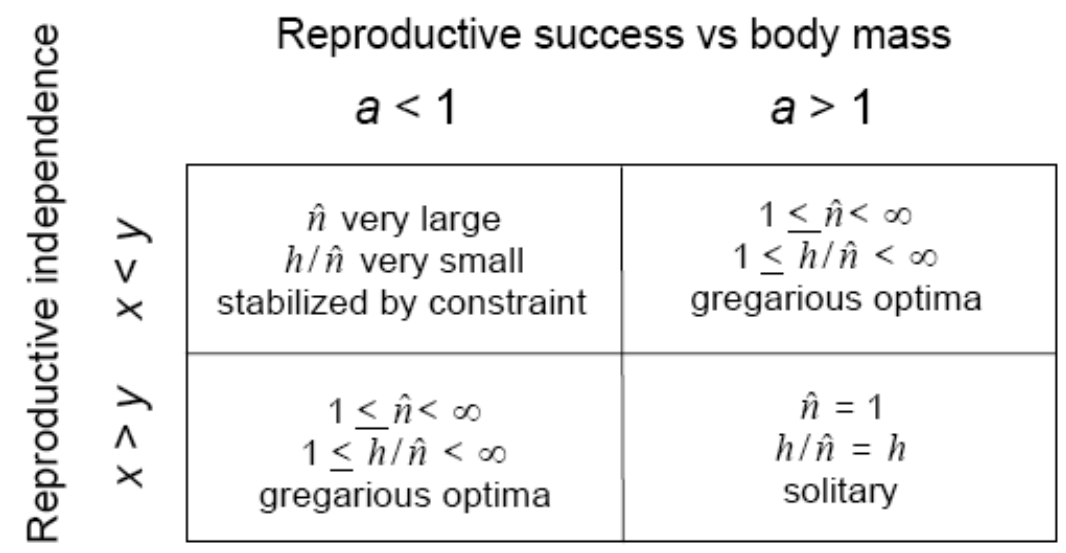

Fig. (3). Model outcomes depending on magnitudes of the exponents that determine fitness. With $a<1$, a female's ability to oviposit into hosts increases sub-proportionally with body mass, whereas for $a>1$ this ability increases supra-proportionally with body mass. Exponents $x$ and $y$ express the respective effects of brood size and body mass on the temporal variability of reproductive success along a lineage sequence. When $x<y$ and $a>1$ or when $x>y$ and $a<1$, the model generates optimal brood size and body mass for "gregarious" broods (multiple individuals). When $a>1$ and $x>y$, fitness is maximized with "solitary" broods consisting of a single offspring. And when $a<1$ and $x<y$, fitness increases with brood size, implying that some constraint on body mass must ultimately set the brood size and body mass. 
identical individuals emerging from the same host caterpillar. Typical values of $x$ might thus fall in the interval $[-0.5,0]$. Effects of body size on independence of reproductive success also seem likely to be small in absolute value, though even the sign of parameter $y$ is unclear. This exponent could be positive or negative, depending on dispersal mechanisms and other details of reproduction, none of which seems likely to be particularly intense. We supposed that $y$ lies in the interval $[-0.3,0.3]$.

Exploitation and vulnerability effects associated with the exponent $a$ are particularly challenging to estimate (Bennett and Hoffman 1998), but a better strategy may be estimating the relationship between female body mass and relative number of hosts likely to be parasitized. For pro-ovigenic parasitoids like the Encyrtidae, females have all of their eggs at eclosion, and particularly for species like Copidosoma bakeri that generally inserts only one egg per host, this egg load should be a good rough indication of expected total oviposition success. In the absence of suitable data from $C$. bakeri, we estimated the data for tibia length vs egg load for female C. floridanum at eclosion from Fig. (5A) of Ode and Strand (1995). We then took logarithms of both variables and conducted a model II regression to estimate the slope and $95 \%$ confidence interval. Under the assumption that body mass is proportional to the cube of tibia length, we divided these values by 3 , producing a slope for egg load vs body mass of $1.26\left(95 \% \mathrm{CI}=[0.94,1.70]\right.$, with $\left.r^{2}=0.47\right)$. We used model II regression to obtain an unbiased slope estimate for this allometric relationship (see Warton et al. 2006). We thus expected $a$ for Copidosoma to exceed 1 and lie near the estimated slope.

The independence coefficient $b$ could not be directly estimated and was therefore set to a magnitude that produced an optimal brood size near values observed in recent empirical work (approximately 1800 female wasps per brood in female C. bakeri-Saeki et al. 2009). Despite this free parameter, the model's behavior was strongly constrained by its

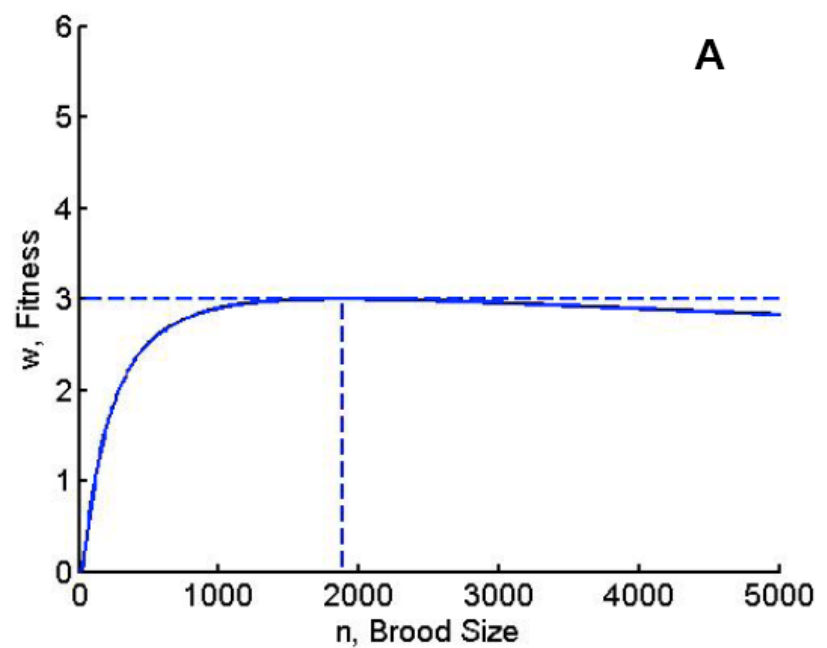

structure and the relevant ranges of the other parameters. For example, while it seems plausible for Copidosoma that $a>1$ and $y>x$ from the arguments above, reversal of both inequalities for this taxon seems highly unlikely, and reversing one but not the other is inconsistent with the existence of a finite optimal brood size greater than 1 for these wasps. We therefore investigated primarily $a>1$ and $y$ $>x$ in the sensitivity analysis.

The unimodal fitness function for representative parameter magnitudes is shown in Fig. (4A), and an alternative parameterization with $a<1$ and $x>y$ that might apply to other taxa is shown in Fig. (4B).

\section{Sensitivity to Parameter Magnitudes}

Much about the sensitivity of $\hat{n}$ to parameter magnitudes is apparent by inspection of equations (6) and (7). These equations imply respectively that optimal brood size $\hat{n} \propto h^{\frac{y}{y-x}}$ and that optimal body mass $\frac{h}{\hat{n}} \propto h^{\frac{x}{x-y}}$, relationships that can be compared directly with data, as we do below. When $x=0, h / \hat{n}$ is independent of $h$, as predicted by some models (Parker and Courtney 1984, Waage and Godfray 1985); but $h / \hat{n}$ depends on $h$ when brood size influences the independence of reproductive success among brood members (i.e. when $x \neq 0$ ). Moreover, when $x<0$ and $y>0$, as we expect for Copidosoma, optimal body mass $h / \hat{n}$ increases with host mass $H(=k h)$. Note that when either $x<0$ and $y>0$ or $x>0$ and $y<0$, both body mass and brood size increase with host mass. This joint increase is predicted by some other models (Skinner 1985, Mayhew and Glaizot 2001, Sakai and Harada 2001; but none of these adequately depicts the general relationships of interest here), and the pattern is often observed empirically (e.g. Opp and Luck 1986, Hardy et al. 1992, Mayhew and Hardy 1998). Fig. (5)

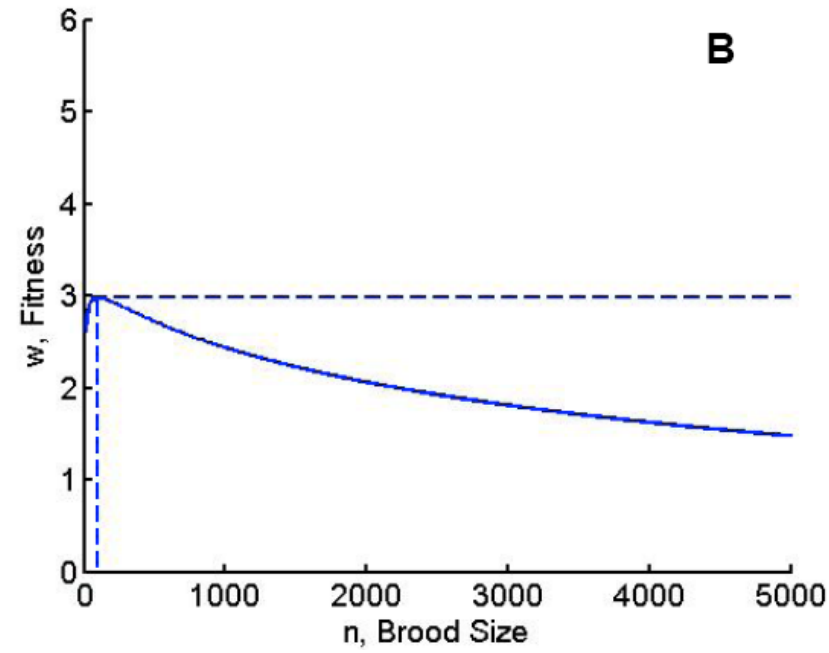

Fig. (4). Brood fitness vs. brood size for females of a hypothetical polyembryonic wasp with single-sex broods. The optimal brood size $\hat{n}$, indicated by the vertical dashed line; the horizontal dashed line shows the maximal brood fitness $w(\hat{n}, h)$. The brood mass $h$ is the proportion of host mass available to the parasitoid, $h / \hat{n}$ is the optimal body size, and $\hat{i}$ indicates the degree of reproductive independence. A. Parameter magnitudes are $a=1.25, b=38.5, c=1, h=1, k=30, x=-0.3$, and $y=0.3$; optima are $\hat{n}=1885, h / \hat{n}=0.531 \times 10^{-3}$, and $w(\hat{n}, h)=3.0$. B. Parameter magnitudes are $a=0.8, b=0.167, c=1, h=1, k=2.3, x=0$, and $y=-0.3$; optima are $\hat{n}=102, h / \hat{n}=9.80 \mathrm{x} 10^{-3}$ and $w(\hat{n}, h)=3.0$. 
illustrates the dependence of $\hat{n}$ and $h / \hat{n}$ on brood mass $h$ (and thus on host mass $H$ through their proportional relationship).

For $a>1$ and $y>x$, optimal brood size $\hat{n}$ increases with the magnitude of $y-x$ (as long as $y-x<e$ ) and with $c^{2}$ (equation (6)), underscoring the importance of bet hedging in enlarging brood size and diminishing body mass. In this

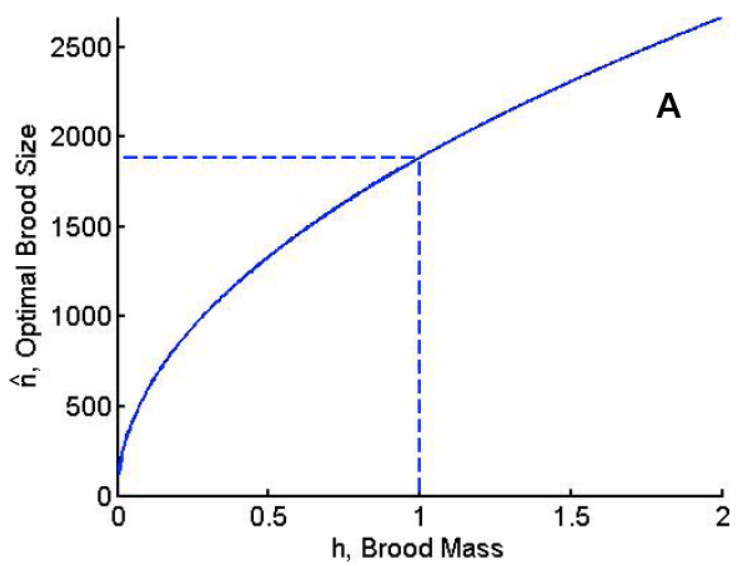

case, since $a>1$, the effects on mean fitness of parallel processing, exploitation, and vulnerability, taken together, tend to diminish brood size and enlarge body mass; as noted above, it is the opposition between bet hedging effects acting through the coefficient of variation and these three processes acting through the mean fitness that balance the size-number trade-off. The optima depend strongly on $x$ and $y$ considered

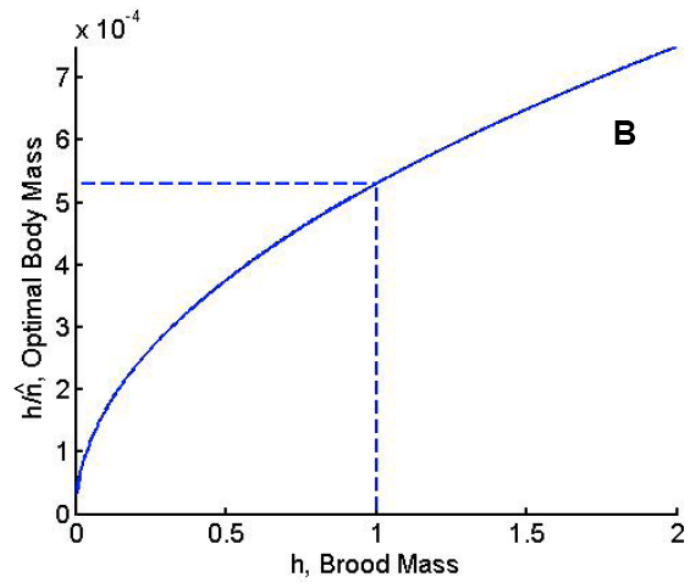

Fig. (5). Optima as functions of brood mass $h$. The dashed lines highlight the standard case with $h=1$. Parameter magnitudes are $a=1.25, b$ $=38.5, c=1, k=30, x=-0.3$, and $y=0.3$. A. Optimal brood size $\hat{n} v s$. brood mass $h$. B. Optimal body mass $h / \hat{n} v s$. brood mass $h$.
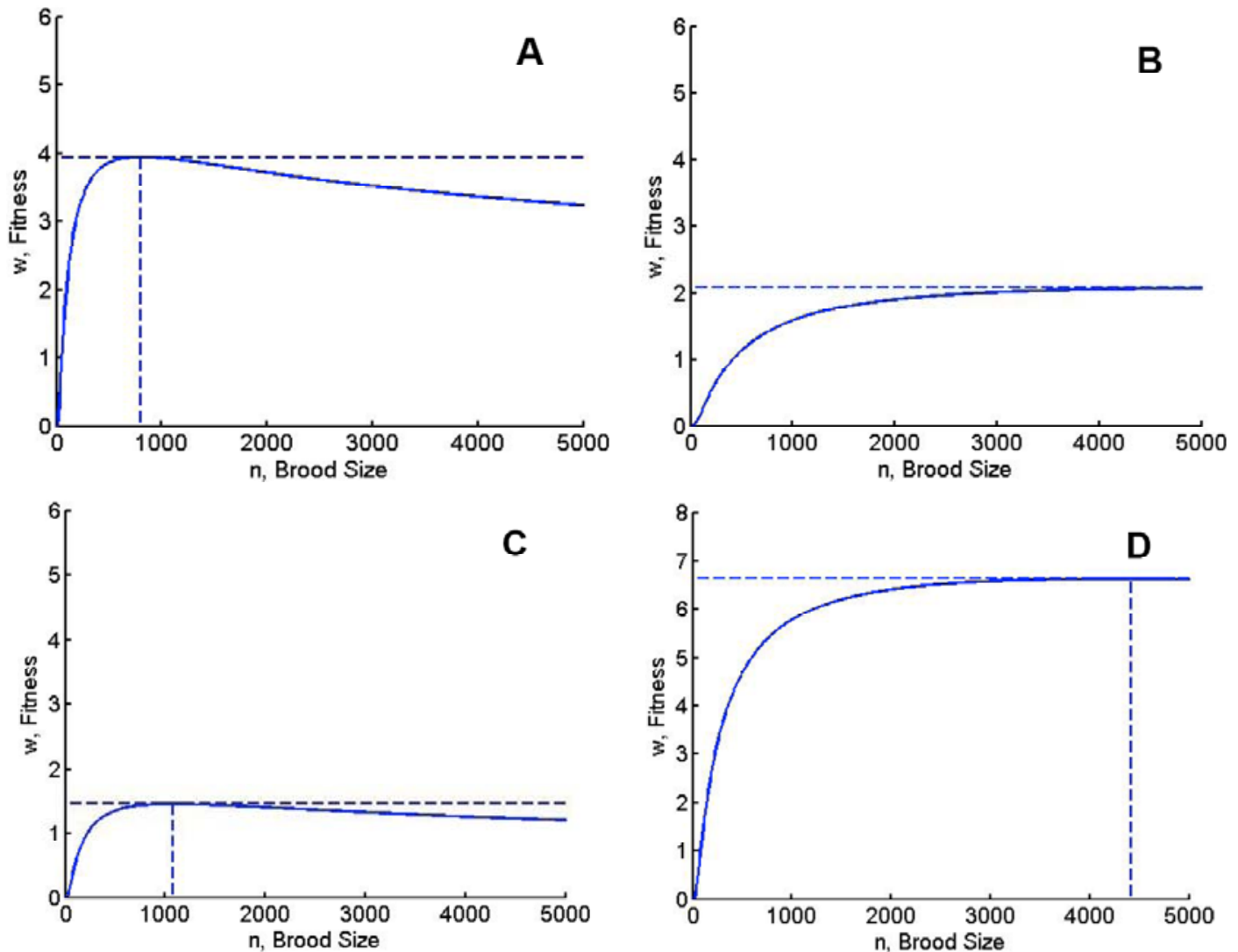

Fig. (6). Brood fitness vs. brood size for females of a hypothetical polyembryonic wasp with single-sex broods. The optimal brood size $\hat{n}$, indicated by the vertical dashed line; the horizontal dashed line shows the maximal brood fitness $w(\hat{n}, h)$. The brood mass $h$ is the proportion of host mass available to the parasitoid, and $h / \hat{n}$ is the optimal body size. A. Parameter magnitudes are $a=1.25, b=38.5, c=1, h=1, k=$ $30, \boldsymbol{x}=\mathbf{- \mathbf { 0 . 4 }}$, and $y=0.3$; optima are $\hat{n}=800, h / \hat{n}=1.25 \times 10^{-3}$, and $w(\hat{n}, h)=3.9$. B. Parameter magnitudes are $a=1.25, b=38.5, c=1, h=$ $1, k=30, \boldsymbol{x}=\mathbf{- \mathbf { 0 . 2 }}$, and $y=0.3$; optima are $\hat{n}=5917, h / \hat{n}=0.169 \times 10^{-3}$, and $w(\hat{n}, h)=2.1$. C. Parameter magnitudes are $\boldsymbol{a}=\mathbf{1 . 3 5}, b=38.5$, $c=1, h=1, k=30, x=-0.3$, and $y=0.3$; optima are $\hat{n}=1076, h / \hat{n}=0.929 \times 10^{-3}$, and $w(\hat{n}, h)=1.5$. D. Parameter magnitudes are $\boldsymbol{a}=\mathbf{1 . 1 5}$, $b=38.5, c=1, h=1, k=30, x=-0.3$, and $y=0.3$; optima are $\hat{n}=4417, h / \hat{n}=0.226 \times 10^{-3}$, and $w(\hat{n}, h)=6.6$. 

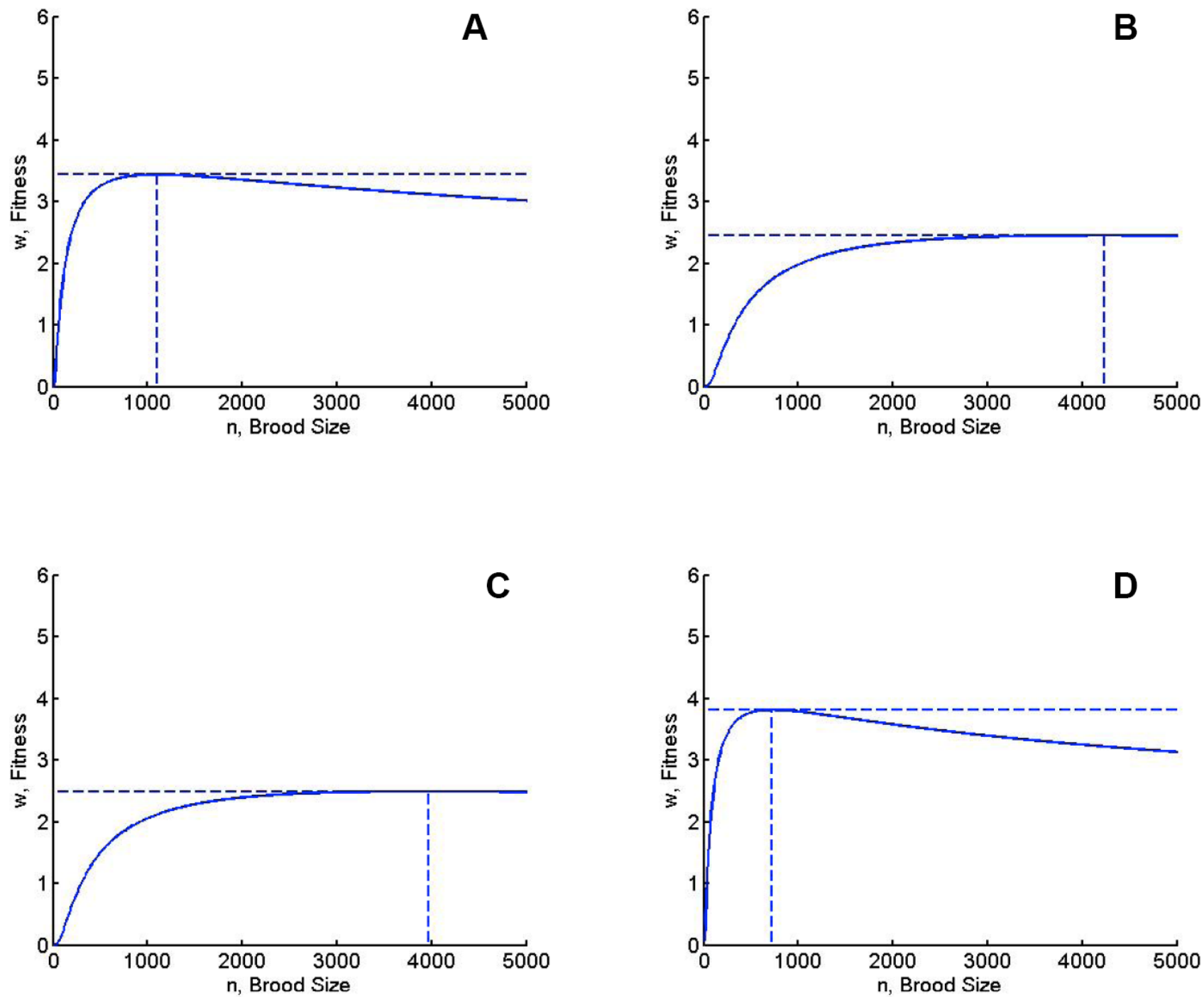

Fig. (7). Brood fitness vs. brood size for females of a hypothetical polyembryonic wasp with single-sex broods. The optimal brood size $\hat{n}$, indicated by the vertical dashed line; the horizontal dashed line shows the maximal brood fitness $w(\hat{n}, h)$. The brood mass $h$ is the proportion of host mass available to the parasitoid, and $h / \hat{n}$ is the optimal body size. A. Parameter magnitudes are $a=1.25, \boldsymbol{b}=\mathbf{2 7 . 8}, c=1, h=1, k=$ $30, x=-0.3$, and $y=0.3$; optima are $\hat{n}=1096, h / \hat{n}=0.912 \times 10^{-3}$, and $w(\hat{n}, h)=3.4$. B. Parameter magnitudes are $a=1.25, \boldsymbol{b}=\mathbf{6 2 . 5}, c=1, h$ $=1, k=30, x=-0.3$, and $y=0.3$; optima are $\hat{n}=4235, h / \hat{n}=0.236 \times 10^{-3}$, and $w(\hat{n}, h)=2.6$. C. Parameter magnitudes are $a=1.25, b=$ $38.5, c=1.25, h=1, k=30, x=-0.3$, and $y=0.3$; optima are $\hat{n}=3967, h / \hat{n}=0.252 \times 10^{-3}$, and $w(\hat{n}, h)=2.5$. D. Parameter magnitudes are $a=1.25, b=38.5, \boldsymbol{c}=\mathbf{0 . 7 5}, h=1, k=30, x=-0.3$, and $y=0.3$; optima are $\hat{n}=723, h / \hat{n}=1.38 \times 10^{-3}$, and $w(\hat{n}, h)=3.8$.

separately (Figs. (6A and 6B); sensitivity to changes in the magnitude of $y$ are identical to those shown for $x$ ) and on the exponent $a$ (Figs. 6C and 6D), which potentiates the advantages of larger body mass, reducing $\hat{n}$ and thus the spreading of reproductive risk, thereby diminishing fitness $w$. The closer that the exponent $a$ is to 1 , the more strongly $\hat{n}$ and $h / \hat{n}$ respond to the magnitude of $a$ (Figs. 6C and 6D).

Optimal brood size $\hat{n}$ and body mass $h / \hat{n}$ also respond strongly to the independence coefficient $b$ (Figs. 7A and 7B) and to the coefficient of variation in reproductive success within generations $c$ (Figs. 7C and 7D). Greater variation in $b$ and $c$ emphasizes bet hedging and thus increases $\hat{n}$ at the expense of $h / \hat{n}$.

For the case with $a<1$ and $x>y$, the parameters have effects opposite to those for $a>1$ and $y>x$, though the magnitudes of the effects are the same. As previously indicated, with $a<1$ and $x>y$, bet hedging tends to diminish brood size and enlarge body mass, whereas the three effects on mean fitness enlarge brood size and diminish body mass.

\section{Comparison with Published Data}

In a recent article, Saeki et al. (2009) presented results of empirical work examining the size-number trade-off in Copidosoma bakeri. They were able to document the tradeoff and the mechanisms used by the developing broods to balance the trade-off differently for male and female broods. These authors emphasized the importance of using models to sharpen our understanding of how post-emergence selection pressures might account for the balance achieved. This is the motivation for the present study.

Of particular interest are the results of Saeki et al. (2009) on the relation to host mass $H$ of both brood size $n$ and body 

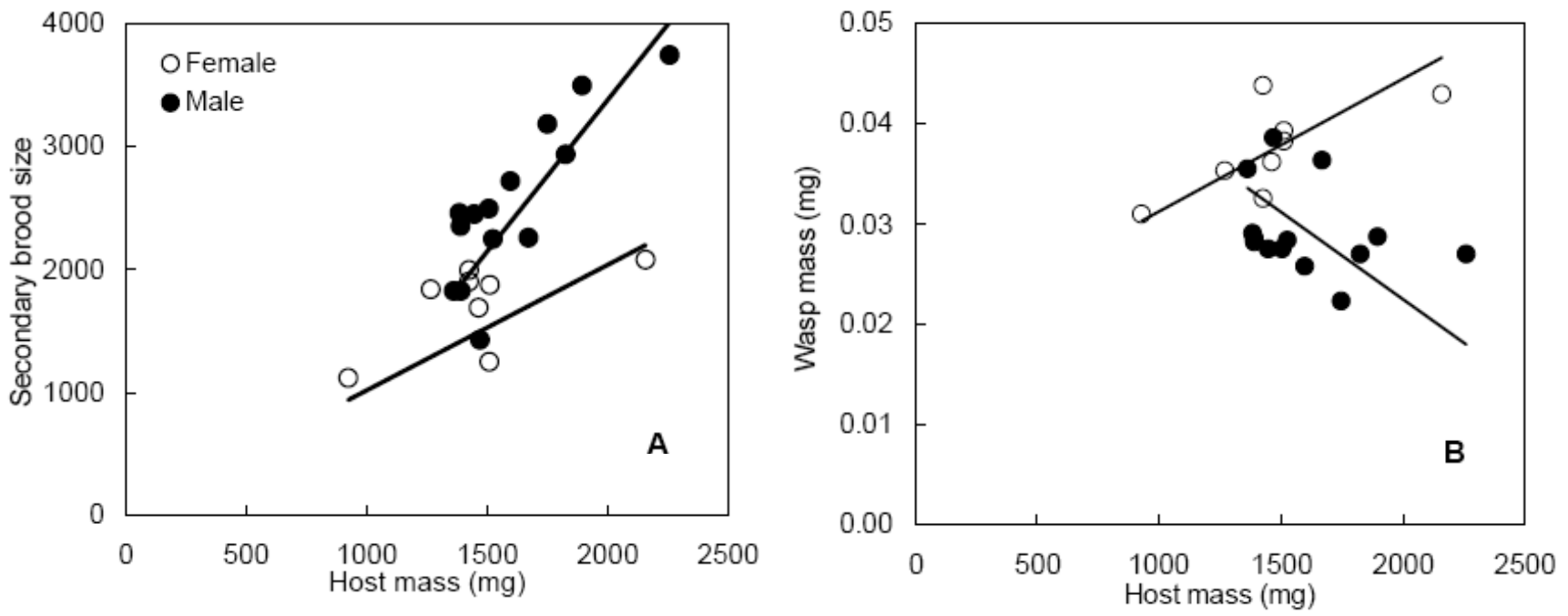

Fig. (8). Brood size vs. host mass (A) and body mass vs host mass (B) of Copidosoma bakeri at emergence from caterpillars of the noctuid moth Agrostis ipsilon (Saeki et al. 2009, reproduced in modified form with permission). Wasp mass is the dry weight of the entire brood; host mass is the maximum wet weight late in the final $\left(6^{\text {th }}\right)$ stadium. Equations for the lines, obtained by model II regression, are $(\mathrm{A}) n=$ $1.02 H+224$ (female broods) and $n=2.46 H-1540$ (male broods) and (B) $h / n=0.0000132 H+0.0181$ (female broods) and $h / n=-$ $0.0000174 H+0.0572$ (male broods).

mass $h / n$. Brood mass $h$ was shown to be proportional to host mass $H$ across broods, and the empirical relationships of Fig. (8) were obtained. The general consistency of Figs. (5A and 8A) and of Figs. (5B and 8B) for females (but not males) is striking. If, as we expect, $a>1$ and $x<0$, then the pattern of data in Fig. (8) suggest that $|x| \approx|y|$ and that $y>0$, producing the apparently sub-proportional increases in both $\hat{n}$ and $h / \hat{n}$ with $H$ (and thus with $h$ ) implied under these conditions by equations (6) and (7). We emphasize, however, that the patterns for females in Fig. (8) are based on relatively few broods. Moreover, while both the slope and intercept of the regression line for female broods in Fig. (8B) are significantly positive, and the trends in Fig. (8A) are similar, neither the slope nor the intercept of the corresponding line in Fig. (8A) differs significantly from zero (Saeki et al. 2009).

\section{DISCUSSION}

We have constructed and analyzed a simple life-history model to show that the balancing of the size-number tradeoff in clonal broods may in some cases be explained as a compromise between two types of fitness effects. Bet hedging can increase fitness by spreading reproductive risk within the brood, thus reducing variation in reproductive success among generations. Other components of reproduction-including the total number of females in search of hosts, their rate of finding them, and their ability to survive while doing so-determine the mean or expected reproductive success within a generation.

Two of the model outcomes indicated in Fig. (3) are "gregarious" optima, meaning optimal brood size and body mass consistent with broods of multiple individuals. Here, both brood size and body mass can both depend on resource level (and thus on brood mass and host mass). For the other two outcomes, however, resource level can only influence one of these brood characteristics. When $a>1$ and $x>y$, the result is a "solitary" offspring; in this case resource level can only make this single individual larger or smaller. When $a<$ 1 and $x<y$, fitness increases with brood size; a lower limit on body mass may result for example from desiccation risk through increasing surface-to-volume ratio or from the inability of tiny wasps to penetrate host eggs. In this case, resource level (brood and host mass) will be linked to brood size rather than body mass.

For optima in the absence of bet hedging effects acting through brood size (when $x=0$ ) or when brood characteristics are stabilized by constraint, the model predicts the production of broods that vary only in size and not in body mass of offspring across resource levels (consistent with models of Parker and Courtney 1984 and Waage and Godfray 1985). Under other conditions for the optimal outcomes, the model can generate the more typical empirical pattern that both brood size and body mass increase with resources (Opp and Luck 1986, le Masurier 1987, Hardy et al. 1992, Ode et al. 1996, Mayhew 1998, Mayhew and Hardy 1998), in particularly close accord with a recent empirical study of the polyembryonic parasitoid Copidosoma bakeri (Saeki et al. 2009). Still other patterns of response to resource levels can be generated by the model for the situations with optima (e.g. brood size or body mass increases supra-proportionally with resource level, while the other brood characteristic being traded off actually decreases with increasing resources).

Parasitic wasps account for about $20 \%$ of all insect species (Traynor and Mayhew 2005) and have proven especially valuable for life-history analysis (Godfray 1994). Polyembryonic species give rise to the largest broods among parasitoids (Trainer and Mayhew 2005). This is probably because producing a large brood represents a negligible cost to the mother and because sibling competition that would otherwise select for larger body size during development is reduced by the high degree of intra-brood relatedness. Clonal broods entirely eliminate sibling competition and produce a 
gender-specific size-number balance that keeps male-female interactions during development (e.g. see Ode and Strand 1995) from complicating the interpretation of results. The clonal brood itself, under the constraint of available host mass, balances the trade-off to maximize total reproductive success of the brood, a balance presumably shaped by generations of selection on ancestral post-emergence adults. Though perhaps conceptually simpler than other systems for which the size-number trade-off is an important issue, the phenomena at work in the clonal polyembryonic case may provide a conceptual foundation (e.g. Fig. 1) onto which complexities like interactions between genotypes and sexes may be added to good effect. In the typical genetically mixed monembryonic case, the mother determines by oviposition at least the upper limit of the eventual brood size, provides resources, and determines the clutch sex ratio. But whether the resulting brood at independence from the mother approaches her optimal number, size, and sex distribution of offspring - or resembles an optimum for any of the offspring themselves-is a far more complex question than the one addressed here.

Few previous models have attempted to explain the commonly observed positive relationship between the amount of resources provided to a developing brood and the body mass of offspring. Skinner (1985) recognized a time trade-off between present and future reproduction that leads to larger offspring with larger hosts only when comparing between populations, where the expected size of the next host to be found is population-specific. This results in more eggs per host but more resources available per individual offspring in populations having larger hosts overall.

Mayhew and Glaizot (2001) note that Skinner's mechanism cannot explain the within-population data. They argue instead that contest competition increasing in intensity for the larger broods associated with larger hosts is likely to reduce survival and allow the survivors access to more resources per individual, producing the positive host mass $v s$. body mass correlation. These authors also postulate that the same trend can arise for the special case of wasps that are synovigenic (i.e. eggs are matured continuously during adult life) and incur greater time delays at the host when more eggs must be first matured and then laid. There is also a substantial plant literature on the size-number trade-off in general and the relation between resources provided to offspring and offspring size in particular (e.g. see Venable 1992 and Hendry et al. 2001). A recent model by Sakai and Harada (2001), the terminal-stream-limitation model, generates a positive relation between seed size and availability of maternal resources (or maternal mass), where optimal seed size depends on a balance between size-related resource extraction rate and maintenance respiration rate.

In contrast to these more specialized models based primarily on physiological constraints and competitive effects, our approach addresses the establishment of a balance between body mass and brood size. This balance is based on the way that these characteristics influence components of reproductive success to determine fitness. Our model presents a broader picture of the major factors likely to be involved in the trade-off, but perhaps at the expense of introducing parameters and functions more difficult to quantify and fully assess.
Our study thus highlights some issues that require attention in future work. Much of the analysis relies heavily on power functions to express key relationships. Functions of this form are widely used in life-history studies and more broadly to depict allometric relationships, but data patterns inconsistent with power functions are well documented (e.g. see King 1987, Kazmer and Luck 1995). For cases in which power functions seem appropriate, it should be possible to get a better fix on the magnitude of the exponent $a$ through direct investigation of vulnerability and exploitation-at least in some systems where field investigation (e.g. see Kazmer and Luck 1995 and Bennett and Hoffmann 1998) or perhaps greenhouse studies are feasible. Those analyses might instead indicate more appropriate functions that better describe those relationships. Ideally, comparative work could examine the magnitude of $a$ (or the parameters of other empirically documented functions) across taxa to identify situations in which mean fitness is expected to increase or decrease with brood size or body mass. Particularly challenging would be obtaining defensible magnitudes of the exponents $x$ and $y$. A better understanding at least of the signs of these parameters if not their magnitudes might be approached through a combination of spatially explicit modeling, field sampling, and microhabitat monitoring.

We have not explicitly addressed the size-number tradeoff for male broods in the analysis above, expecting males to use rather different strategies from those of females. Among Encyrtid wasps, some males appear to mate primarily with sisters from the same mixed-sex brood (Strand 1989), some apparently disperse individually in search of females (Hardy 1994), some may seek emerging broods to gain multiple matings (Godfray 1994), and some may swarm, perhaps as a clonal group, to attract females (Nadel 1987). It seems likely that male mating strategy is a plastic trait, depending on characteristics of the brood, proximity to females, weather, and landscape. Reproductive success may be more weakly linked to body size in males, and males can mate successfully with a wide size range of larger-to-smaller females (YS, unpublished observations). Exploiting this size flexibility, males may generally tend to have a smaller body size than females to maximize their brood size, subject to the constraint of being just large enough to mate the females. In other words, parallel processing effects that increase the total reproductive success with the brood size should remain important for males. This might help explain why additional resources obtained from larger hosts result in larger brood size but not larger body mass in C. bakeri (Fig. 8; Saeki et al. 2009; but see Strand 2000 on C. floridanum).

\section{ACKNOWLEDGEMENTS}

We thank the Crowley lab group at the University of Kentucky, the research group of Nico Michiels at the University of Tübingen, Germany, and the participants at two conferences (Center for Ecology, Evolution and Behavior at the University of Kentucky, May, 2008; and Analogies in the Evolution of Gender Expression and Sexual Strategies in Animals and Plants [ASAP] in Stuttgart, Germany, September, 2008) for comments and suggestions on this project. We also thank Volker Grimm, Paul Ode, and an anonymous reviewer for helpful input that improved the manuscript. We acknowledge grant support from Sigma Xi 
and from the Gertrude Flora Ribble Fund, University of Kentucky Department of Biology.

\section{REFERENCES}

Askew, RR (1968) Considerations on speciation in Chalcidoidea (Hymenoptera). Evolution, 22, 642-5.

Bennett, DM \& Hoffmann, AA (1998) Effects of size and fluctuating asymmetry on field fitness of the parasitoid Trichogramma carverae (Hymenoptera: Trichogrammatidae). Journal of Animal Ecology, 67, 580-91.

Bulmer, M (1994) Theoretical Evolutionary Ecology. Sinauer Assoc. Inc., Sunderland, MA, USA

Copland, MJW \& Askew, RR (1977) An analysis of the chalcidoid (Hymeoptera) fauna of a sand-dune system. Ecological Entomology, 2, 27-46.

Crowley, PH (1977) Spatially distributed stochasticity and the constancy of ecosystems. Bulletin of Mathematical Biology, 39, 157-66.

Crowley, PH (2000) Sexual dimorphism with female demographic dominance: age, size, and sex ratio at maturation. Ecology, 81, 2592-605.

Crowley, PH, Saeki, Y \& Switzer, PV (2009) Evolutionarily stable oviposition and sex ratio in parasitoid wasps with single-sex broods. Ecological Entomology, 34, 163-75.

Fox, C W \& Czesak, ME (2000) Evolutionary ecology of progeny size in arthropods. Annual Review of Entomology, 45, 341-69.

Glenn, DC \& Hoffman, AA (1997) Developing a commercially viable system for biological control of Epiphyas postvittana (Lepidoptera: Tortricidae) in grapes using endemic Trichogramma (Hym: Trichogrammatidae). Journal of Economic Entomology, 90, 37082.

Godfray, HCJ (1987) The evolution of clutch size in parasitic wasps. American Naturalist, 129, 221-233.

Godfray, HCJ (1994) Parasitoids: Behavioral and Evolutionary Ecology. Princeton University Press, Princeton, NJ, USA.

Godfray, HCJ \& Parker, GA (1991) Clutch size, fecundity and parentoffspring conflict. Philosophical Transactions of the Royal Society of London B, 332, 67-79.

Hardy, ICW (1994) Sex ratio and mating structure in the parasitoid Hymenoptera. Oikos, 69, 3-20.

Hardy, ICW, Griffiths, NT \& Godfray, HCJ (1992) Clutch size in a parasitoid wasp: a manipulation experiment. Journal of Animal Ecology, 61, 121-9.

Hendry, AP, Day, T, \& Cooper, AB (2001) Optimal size and number of propagules: allowance for discrete stages and effects of maternal size on reproductive output and offspring fitness. The American Naturalist, 157, 387-407.

Ivanova-Kasas, OM (1972) Polyembryony in insects. In: Counce, SJ \& Waddington, CH (eds.) Developmental Systems, Insects, Academic Press, London, Vol 2: 243-71.

Kazmer, DJ \& Luck, RF (1995) Field tests of the size-fitness hypothesis in the egg parasitoid Trichogramma pretiosum. Ecology, 76, 412-25.

King, BH (1987) Offspring sex ratios in parasitoid wasps. Quarterly Review of Biology, 62, 367-96.

Lack, D (1947) The significance of clutch size. Ibis, 89, 309-52.

Lewontin, RC \& Cohen, D (1969) On population growth in a randomly varying environment. Proceedings of the National Academy of Sciences USA, 62, 1056-60.

le Masurier, AD (1987) A comparative study of the relationship between host size and brood size in Apanteles spp. (Hymenoptera: Braconidae). Ecological Entomology, 12, 383-93.
Mayhew, PJ (1998) Offspring size-number strategy in the bethylid parasitoid Laelius pedatus. Behavioral Ecology, 9, 54-9.

Mayhew, PJ \& Glaizot, O (2001) Integrating theory of clutch size and body size evolution for parasitoids. Oikos, 92, 372-6.

Mayhew, PJ \& Hardy, ICW (1998) Nonsiblicidal behavior and the evolution of clutch size in bethylid wasps. The American Naturalist, 151, 409-24.

Morris, WF, Pfister, CA, Tuljapurkar, S, Haridas, CV, Boggs, CL, Boyce, MS, Bruna, EM, Church, DR, Coulson, T, Doak, DF, Forsyth, S, Gaillard, J-M, Horvitz, CC, Kalisz, S, Kendall, BE, Knight, TM, Lee, CT \& Menges, ES (2008) Longevity can buffer plant and animal populations against changing climatic variability. Ecology, $89,19-25$.

Nadel, H (1987) Male swarms discovered in Chalcidoidea (Hymenoptera: Encyrtidae, Pteromalidae). Pan Pacific Entomologist, 63, 242-6.

Ode, PJ, Antolin, MF \& Strand, MR (1996) Sex allocation and sexual asymmetries in intra-brood competition in the parasitic wasp Bracon hebetor. Journal of Animal Ecology, 65, 690-700.

Ode, PJ \& Strand, MR (1995) Progeny and sex allocation decisions of the polyembryonic wasp Copidosoma floridanum. Journal of Animal Ecology, 64, 213-24.

Opp, SB \& Luck, RF (1986) Effects of host size on selected fitness components of Aphytis melinus and A. lingnanensis (Hymenoptera: Aphelinidae). Annals of the Entomological Society of America, 79, 700-4.

Parker, GA \& Courtney, SP (1984) Models of clutch size in insect oviposition. Theoretical Population Biology, 26, 27-48.

Roff, DA (2002) Life history evolution. Sinauer Associates, Inc., Sunderland, MA, USA

Saeki, Y, Crowley, PH, Fox, CW \& Potter, DA (2009) A sex-specific sizenumber trade-off in clonal broods. Oikos, 34, 163-175.

Sakai, S \& Harada, Y (2001) Why do large mothers produce large offspring? Theory and a test. The American Naturalist, 157, 34859

Skinner, SW (1985) Clutch size as an optimal foraging problem for insects. Behavioral Ecology and Sociobiology, 17, 231-8.

Smith, CC \& Fretwell, SD (1974) The optimal balance between the size and number of offspring. The American Naturalist, 108, 499-506.

Stearns, SC (1992) The Evolution of Life Histories. Oxford University Press, Oxford, UK

Strand, MR (1989) Clutch size, sex ratio and mating by the polyembryonic encyrtid Copidosoma floridanum (Hymenoptera: Encyrtidae). Florida Entomologist, 72, 32-42.

Strand, MR (2000) Developmental traits and life-history evolution in parasitoids. In: Hochberg, ME \& Ives, AR (Eds.) Parasitoid Population Biology. Princeton University Press, Princeton, NJ USA, 139-62.

Traynor, RE \& Mayhew, PJ (2005) A comparative study of body size and clutch size across the parasitoid Hymenoptera. Oikos, 109, 305-16.

Venable, DL (1992) Size-number trade-offs and the variation of seed size with plant resource status. The American Naturalist, 140, 287-304.

Waage, JK \& Godfray, HCJ (1985) Reproductive strategies and population ecology of insect parasitoids. In: Sibly, RM \& Smith, RH (Eds.) Behavioural Ecology. Blackwell Scientific Publishers, Oxford, UK, 449-70.

Warton, DI, Wright, IJ, Falster, DS \& Westoby, M (2006) Bivariate linefitting methods for allometry. Biological Review, 81, 259-91.

West, SA, Flanagan, KE \& Godfray, HCJ (1996) The relationship between parasitoid size and fitness in the field, a study of Achrysocharoides zwoelferi (Hymenoptera: Eulophidae). Journal of Animal Ecology, $65,631-9$. 